\title{
Counterexample-Guided Abstraction Refinement for the Analysis of Graph Transformation Systems ${ }^{\star}$
}

\author{
Barbara König and Vitali Kozioura \\ Institut für Formale Methoden der Informatik, Universität Stuttgart, Germany \\ \{koenigba, koziouvi\}@fmi.uni-stuttgart.de
}

\begin{abstract}
Graph transformation systems are a general specification language for systems with dynamically changing topologies, such as mobile and distributed systems. We propose a counterexample-guided abstraction refinement technique which is based on the over-approximation of graph transformation systems (GTS) by Petri nets. We show that a spurious counterexample is caused by merging nodes during the approximation. We present a technique for identifying these merged nodes and splitting them using abstraction refinement, which removes the spurious run. The technique has been implemented in the Augur tool and experimental results are discussed.
\end{abstract}

\section{Introduction}

In the last years verification techniques based on counterexample-guided abstraction refinement [8] have been very successful. The idea behind this approach is to start with a coarse initial over-approximation and to refine this abstraction by eliminating spurious counterexamples. The technique has been used successfully in several tools such as SLAM [6], BLAST [10] or MAGIC 7].

Abstraction is also important for graph structures that can arise in several applications, for instance as evolving pointer structures on the heap, as object graphs or as networks with mobile processes. So far, little work has been done in this area concerning abstraction refinement. We are only aware of [12] where models of a 3-valued logics representing pointer structures are refined in the framework of shape analysis [16] by generating new instrumentation relations.

Here we are working in a different framework where we are using graph transformation systems (GTS) - instead of 3-valued logics — in order to represent and transform graph structures. Graph transformation systems are an expressive and useful specification formalism, allowing to describe dynamic properties of concurrent and distributed systems [15]. They can be used to model systems such as pointer structures, object-oriented languages and mobile processes.

In this paper the technique of counterexample-guided abstraction refinement is applied to the verification of graph transformation systems. Our approach is based on a (partial order) technique that approximates GTSs by Petri nets via

\footnotetext{
* Research supported by DFG project SANDS and SFB 627 (NEXUS).
} 
an unfolding construction 3. More specifically, in this approach a finite overapproximation called Petri graph is constructed, which consists of a graph and a Petri net having the edges of the graph as places. The important property of the approximation obtained in this way is that each graph reachable from the start graph in the GTS can be mapped, by merging some of its nodes, to a reachable marking of the over-approximating Petri net. On the other hand there may be some markings reachable in the obtained Petri graph, which have no counterpart in the original GTS. The sequence of events in the approximation leading to such a graph is called a spurious run.

In our case spurious runs are caused by the merging of graph nodes in the construction of the over-approximation. This is similar to the concept of summary nodes in shape analysis [16]. This paper describes how to construct a more exact over-approximation by separating merged nodes for which these spurious runs disappear. This procedure can be performed repeatedly for any number of spurious runs.

We believe that the technique of identifying the reason for the spurious run is independent of the abstraction mechanism used in this paper and could also be used in other frameworks dealing with approximations of graph structures.

The techniques presented here are implemented as an extension of the tool Augur 1. The experimental part of the paper compares this approach with an already existing abstraction refinement technique which reduces the number of spurious examples by constructing an over-approximation which is exact up to some pre-defined depth [5]. It is shown experimentally that counterexampleguided abstraction refinement is faster and produces smaller Petri graphs.

A long version of this paper is available as a technical report [1].

\section{Basic Notions}

In this section we describe the notions of hypergraph, GTS, Petri net and Petri graph and also show in an informal way how to construct over-approximating Petri graphs.

Definition 1 (hypergraphs and hypergraph morphisms). Let $\Lambda$ be a set of labels where each label $l \in \Lambda$ has an arity $\operatorname{ar}(l) \in \mathbb{N}$. A labelled hypergraph $G$ is a tuple $\left(V_{G}, E_{G}, c_{G}, l_{G}\right)$, where $V_{G}$ is a finite set of nodes, $E_{G}$ is a finite set of edges, $c_{G}: E_{G} \rightarrow V_{G}^{*}$ is a connection function and $l_{G}: E_{G} \rightarrow L$ is the labeling function satisfying $\operatorname{ar}\left(l_{G}(e)\right)=\left|c_{G}(e)\right|$ for every $e \in E_{G}$. The nodes are not labelled.

Let $G$ and $G^{\prime}$ be two labelled hypergraphs. A hypergraph morphism (or simply morphism) $\varphi: G_{1} \rightarrow G_{2}$ consists of a pair of total functions $\varphi_{V}: V_{G_{1}} \rightarrow V_{G_{2}}$ and $\varphi_{E}: E_{G_{1}} \rightarrow E_{G_{2}}$ such that for every $e \in E_{G_{1}}$ it holds that $l_{G_{1}}(e)=$ $l_{G_{2}}\left(\varphi_{E}(e)\right)$ and $\varphi_{V}\left(c_{G_{1}}(e)\right)=c_{G_{2}}\left(\varphi_{E}(e)\right)$. A morphism is called edge-bijective (edge-injective) whenever it is bijective (injective) on edges. It is an isomorphism whenever it is bijective on nodes and edges.

Hypergraphs can be rewritten using rules of the following kind.

\footnotetext{
${ }^{1}$ Available from http://www.fmi.uni-stuttgart.de/szs/tools/augur/
} 
Definition 2 (rewriting rule). A rewriting rule $r$ is a triple $(L, R, \alpha)$, where $L$ and $R$ are hypergraphs, called left-hand side and right-hand side respectively and $\alpha: V_{L} \rightarrow V_{R}$ is an injective mapping, indicating how nodes are preserved.

We demand that there are no isolated nodes in the left-hand side $L$ and no isolated nodes in $V_{R}-\alpha\left(V_{L}\right)$. Additionally $E_{L}$ must not be empty.

The first condition says that we abstract from isolated nodes, whereas the second is a standard requirement for unfolding-based techniques, where every rule must be consuming. Note furthermore that we do not consider rules that preserve edges of the left-hand side.

For convenience we will in the following often assume that $\alpha$ is an inclusion denoted by $i d$, which can be enforced by renaming the nodes of the left or righthand side appropriately, and that the node and edge sets of $L$ and $R$ are disjoint otherwise. That is, we demand that $V_{L} \subseteq V_{R}$ and $E_{L} \cap E_{R}=\emptyset$ which implies that the union $L \cup R$ is well-defined.

Given a hypergraph, a rewriting rule and a match of the left-hand side, we can apply this rule and replace the left-hand side by the right-hand side in the following way. Additionally we define a partial morphism $\nu$ from the original graph to the rewritten graph, keeping track of preserved nodes and edges.

Definition 3 (rewriting step). Let $r=(L, R, i d)$ be a rewriting rule. A match of $r$ in a hypergraph $G$ is any morphism $\varphi: L \rightarrow G$ injective on edges. We can apply $r$ to $G$ according to the match $\varphi$ and obtain a new graph $H$, written $G \Rightarrow_{r}$ $H$, which is defined as follows: $V_{H}=V_{G} \uplus\left(V_{R}-V_{L}\right), E_{H}=\left(E_{G}-\varphi\left(E_{L}\right)\right) \uplus E_{R}$ and, defining $\bar{\varphi}: V_{R} \rightarrow V_{H}$ by $\bar{\varphi}(v)=\varphi(v)$ if $v \in V_{L}$ and $\bar{\varphi}(v)=v$ otherwise, the connection and labelling functions are given by $c_{H}(e)=c_{G}(e), l_{H}(e)=l_{G}(e)$ if $e \in E_{G}-\varphi\left(E_{L}\right)$ and $c_{H}(e)=\bar{\varphi}\left(c_{R}(e)\right), l_{H}(e)=l_{R}(e)$ if $e \in E_{R}$.

We also define an injective partial morphism $\nu: G \rightarrow H$ where $\nu_{V}: V_{G} \rightarrow V_{H}$ and $\nu_{E}:\left(E_{G}-\varphi\left(E_{L}\right)\right) \rightarrow E_{H}$ with $\nu(x)=x$ for every node or edge $x$.

A graph transformation system (GTS) $\mathcal{G}=\left(\mathcal{R}, G_{0}\right)$ is a finite set of rules together with a start hypergraph (also called initial graph).

Example: We illustrate the definitions of this chapter with an example describing a firewall system similar to the one introduced in 4. This system contains an (arbitrarily large) set of processes running behind a firewall (safe processes) and one process in a public area (unsafe process). Any number of safe processes $(S P)$ and connected locations $(L)$ can be generated during runtime. The property to verify is that the unsafe process from the public area does not penetrate the firewall. If this situation is detected, rule "Error" will be applied and an edge labelled Error is created.

Fig. 1 and Table 1 depict the initial graph and the rules of the firewall system. A double-headed arrow in a rule means that the rule can be applied in both directions. Numbers close to the nodes indicate the mapping $\alpha$. The private and public areas are connected by the firewall $(F)$, and initially there is one unsafe processes $(U P)$ in the public area. Only safe processes will be generated and the firewall can be crossed in one direction only. Our aim is to show that no reachable graph contains the 0-ary edge Error. 


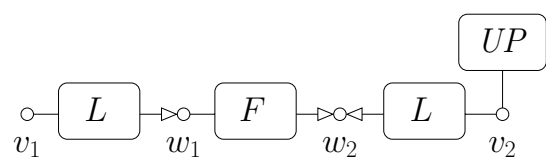

Fig. 1. Initial graph of the firewall system

Table 1. Rules of the firewall system

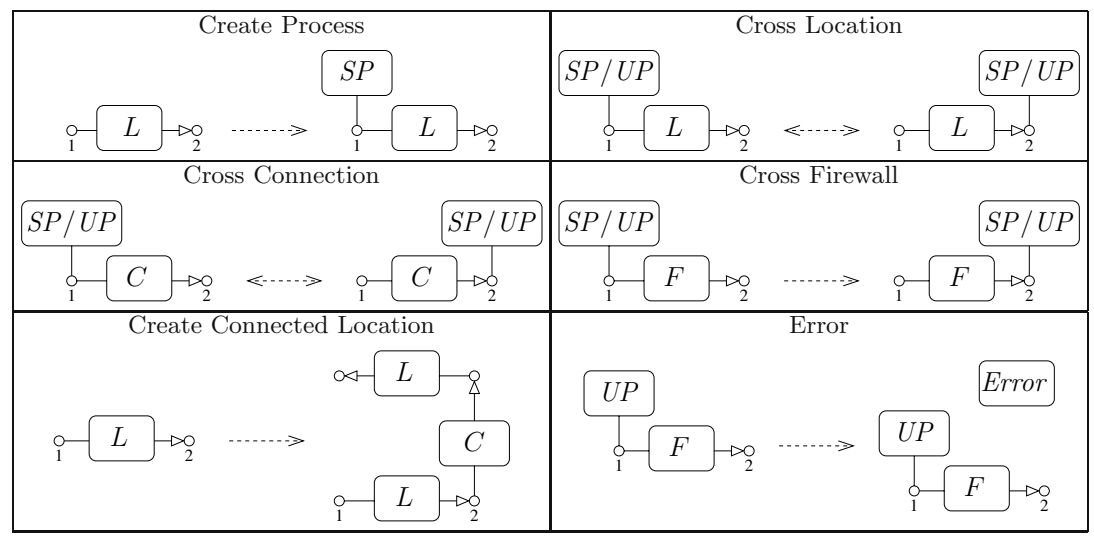

In order to approximate GTSs we will employ Petri nets, which, as multiset rewriting systems, can be seen as a special case of graph rewriting. Petri nets are an easier model than GTS and hence more amenable to analysis. Several algorithms and tools are available for their verification. Furthermore, by approximating with Petri nets we will be able to preserve nice properties of the GTS model, such as locality (state changes are only described locally) and concurrency (no unnecessary interleaving of events) in the approximation.

We will now introduce a notation for Petri nets 2

Definition 4 (Petri net). Let $\Delta$ be a finite set of labels. $A \Delta$-labelled Petri net is a tuple $N=\left(S, T,{ }^{\bullet}(),()^{\bullet}, p\right)$, where $S$ is the set of places, $T$ is a set of transitions, ${ }^{\bullet}(),()^{\bullet}: T \rightarrow S^{\oplus}$ assign to each transition its pre-set and post-set and $p: T \rightarrow \Delta$ assigns a label to each transition. A marked Petri net is a pair $\left(N, m_{N}\right)$, where $N$ is a Petri net and $m_{N} \in S^{\oplus}$ is the initial marking.

\section{Approximated Unfolding}

In this section we will give a short overview of a technique that approximates a graph transformation system by a structure that is both a Petri net and a hypergraph [3, 4, 5].

${ }^{2}$ By $A^{\oplus}$ we denote a multiset over $A$ and for a function $f: A \rightarrow B$ we denote by $f^{\oplus}: A^{\oplus} \rightarrow B^{\oplus}$ its extension to multisets. Furthermore for $m \in A^{\oplus}$ and $a \in A$ we denote by $m(a)$ the multiplicity of $a$ in $m$. 
First we define the notion of Petri graph which will be used to represent an over-approximation for a given GTS. Note that the edges of the graph are at the same time the places of the net and that the transitions are labelled with rules of the GTS.

Definition 5 (Petri graph). Let $\mathcal{G}=\left(\mathcal{R}, G_{0}\right)$ be a GTS. A Petri graph (over $\mathcal{R}$ ) is a tuple $P=(G, N, \mu)$, where $G$ is a hypergraph, $N=\left(E_{G}, T_{N},{ }^{\bullet}(),()^{\bullet}, p_{N}\right)$ is an $\mathcal{R}$-labelled Petri net where the places are the edges of $G$ and $\mu$ associates to each transition $t \in T_{N}$, with $p_{N}(t)=(L, R, i d)$, a hypergraph morphism $\mu(t): L \cup R \rightarrow G$ such that $\bullet^{\bullet}=\mu(t)^{\oplus}\left(E_{L}\right)$ and $t^{\bullet}=\mu(t)^{\oplus}\left(E_{R}\right)$.

$A$ Petri graph for the GTS $\mathcal{G}$ is a pair $(P, \iota)$, where $P=(G, N, \mu)$ is a Petri graph over $\mathcal{R}$ and $\iota: G_{0} \rightarrow G$ is a graph morphism. A marking is reachable (coverable) in Petri graph if it is reachable (coverable) in the underlying Petri net with the multiset $\iota^{\oplus}\left(E_{G_{0}}\right)$ as the initial marking.

We view Petri graphs as symbolic representations of transition systems with graphs as states. Specifically each marking $m$ of a Petri graph $\left(G, N, m_{0}\right)$ can be seen as representation of a graph, denoted by $\operatorname{graph}(m)$, according to the following definition: We take the marked subgraph of $G$ and duplicate each edge as indicated by the marking.

Alternatively one can define $\operatorname{graph}(m)$ as the unique graph $H$, up to isomorphism, such that $H$ has no isolated nodes and there exists a morphism $\psi: H \rightarrow G$, injective on nodes, with $\psi^{\oplus}\left(E_{H}\right)=m$. Furthermore, whenever there exists a morphism $\varphi: G^{\prime} \rightarrow G$ such that $\varphi^{\oplus}\left(E_{G^{\prime}}\right) \leq m$, then there exists an edge-injective morphism $e_{m, \varphi}: G^{\prime} \rightarrow \operatorname{graph}(m)$ such that $\psi \circ e_{m, \varphi}=\varphi$.

In order to obtain a Petri graph approximating a GTS, we first need - as building blocks - Petri graphs that describe the effect of a single rule.

Definition 6 (Petri graph for a rewriting rule). Let $r=(L, R, i d)$ be a rewriting rule. By $P(t, r)=(G, N, \mu)$ we denote a Petri graph with $G=L \cup R$ and $N$ is a net with places $S_{N}=E_{L} \cup E_{R}$ and one transition $t$ such that $p_{N}(t)=r$, $\bullet t=E_{L}$ and $t^{\bullet}=E_{R}$. Furthermore the morphism $\mu(t): L \cup R \rightarrow G$ is the identity.

Given a GTS $\mathcal{G}=\left(\mathcal{R}, G_{0}\right)$ one can construct an over-approximating Petri graph $\mathcal{C}_{\mathcal{G}}$ (also called the covering of $\mathcal{G}$ ), using the following algorithm (see [3]). It starts with a Petri graph $P_{0}$ that consists only of the start graph and computes $\mathcal{C}_{\mathcal{G}}$ iteratively. It is based on an unfolding technique which is combined with over-approximating folding steps which guarantee a finite approximation.

Algorithm 7 (approximated unfolding). We set $P_{0}=\left(G_{0}, N_{0}, m_{0}\right)$, where $N_{0}$ contains no transitions, $m_{0}=E_{G_{0}}$ and let $\iota_{0}: G_{0} \rightarrow G_{0}$ be the identity. As long as one of the following steps is applicable, transform $P_{i}$ into $P_{i+1}$ according to the possibilities given below (where folding steps take precedence over unfolding steps).

Unfolding: Find a rule $r=(L, R, i d) \in \mathcal{R}$ and a match $\varphi: L \rightarrow G_{i}$. Then choose a new transition $t$ and extend $P_{i}$ by attaching $P(t, r)$, i.e., take the disjoint union 
of both Petri graphs and factor through the equivalence $\equiv$ generated by $e \equiv \varphi(e)$ for every $e \in E_{L}$.

Folding: Find a rule $r=(L, R, i d) \in \mathcal{R}$ and two matches $\varphi, \varphi^{\prime}: L \rightarrow G_{i}$ such that $\varphi^{\oplus}\left(E_{L}\right)$ and $\varphi^{\prime \oplus}\left(E_{L}\right)$ are coverable in $N_{i}$ and the second match is causally dependent on the transition unfolding the first match. Then merge the two matches by setting $\varphi(e) \equiv \varphi^{\prime}(e)$ for each $e \in E_{L}$ and factoring through the resulting equivalence relation $\equiv$.

If neither possibility applies the Petri graph $P_{i}$ obtained in the last step is returned. The result is denoted by $\mathcal{C}_{\mathcal{G}}$. In 3 it has been shown that the algorithm always terminates with a result unique up to isomorphism.

In our running example, the constructed over-approximation consists of the hypergraph in Fig. 2 and the Petri net in Fig. 3. (Ignore the highlighted transitions for the moment.) Note that the set of edges of the graph corresponds exactly to the set of places of the net (the correspondence is indicated by giving indices to the labels).

Before we can show in what way Petri graphs can be considered as abstractions of GTSs and before we discuss how they can be analyzed, we first need the definition of an abstract run of a GTS and a notion of correspondence of two abstract runs. Then we can define how Petri graphs can be seen as abstractions of GTSs.

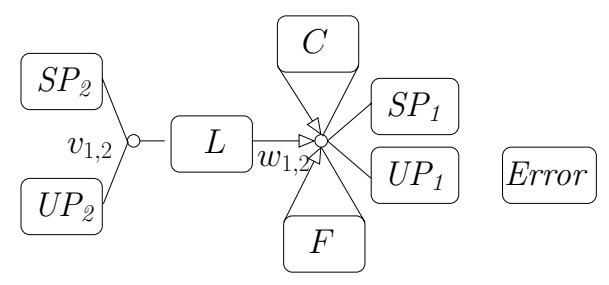

Fig. 2. Hypergraph component of the approximating Petri graph (firewall example)

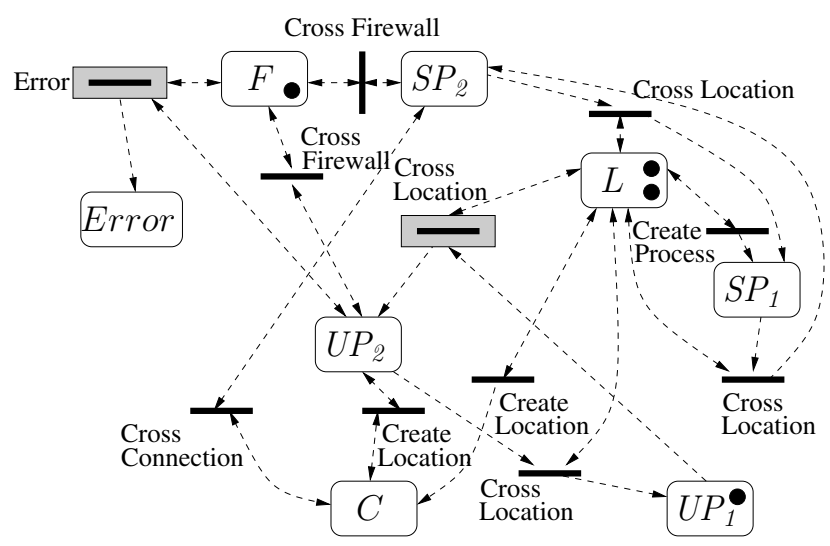

Fig. 3. Petri net component of the approximating Petri graph (firewall example) 
Definition 8 (Abstract run). An abstract run of a $\operatorname{GTS}\left(\mathcal{R}, G_{0}\right)$ is a sequence of hypergraphs $\mathcal{J}=\left(J_{0} \Rightarrow_{r_{1}} J_{1} \Rightarrow_{r_{2}} \ldots \Rightarrow_{r_{n}} J_{n}\right)$, where $r_{i}$ is a rule name, together with morphisms $\varphi_{i}: L_{i+1} \rightarrow J_{i}$ for each $i=1, \ldots, n-1$, where $L_{i}$ is the left-hand side of rule $r_{i} \in \mathcal{R}$.

Note that we do not demand that $J_{i}$ can be derived from $J_{i-1}$ by applying rule $r_{i}$ at match $\varphi_{i}$. In this case $\mathcal{J}$ will be called a real run and we will also use the symbol $\Rightarrow$ instead of $\Rightarrow$.

Let $\mathcal{J}^{\prime}=\left(J_{0}^{\prime} \Rightarrow_{r_{1}} J_{1}^{\prime} \Rightarrow_{r_{2}} \ldots \Rightarrow_{r_{n}} J_{n}^{\prime}\right)$ be another abstract run with morphisms $\varphi_{i}^{\prime}: L_{i+1} \rightarrow J_{i}^{\prime}$ for each $i=1, \ldots, n-1$. We say that $\mathcal{J}^{\prime}$ weakly corresponds to $\mathcal{J}$ (in symbols $\mathcal{J}^{\prime} \ll \mathcal{J}$ ) if for each $i=1, \ldots, n-1$ there exist edge-bijective morphism $\xi_{i}: J_{i}^{\prime} \rightarrow J_{i}$ for $i=0, \ldots, n$. If furthermore the following diagram commutes we say that $\mathcal{J}^{\prime}$ corresponds to $\mathcal{J}$ and write $\mathcal{J}^{\prime} \lll \mathcal{J}$.

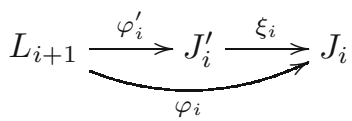

Petri graphs can, as mentioned above, be seen as symbolic representations of graph transition systems and also as representations of sets of abstract runs.

Definition 9 (Abstract runs of a Petri graph). Let $(P, \iota)$ with $P=(G, N, \mu)$ be a Petri graph for a GTS $\left(\mathcal{R}, G_{0}\right)$. Furthermore let $m_{0}\left[t_{1}\right\rangle \ldots\left[t_{n}\right\rangle m_{n}$ be a firing sequence of the net $N$ and let $r_{i}=p_{N}\left(t_{i}\right)$ be the rules corresponding to the transitions. We define morphisms $\varphi_{i}=e_{m_{i},\left.\mu\left(t_{i+1}\right)\right|_{L_{i+1}}}: L_{i+1} \rightarrow \operatorname{graph}\left(m_{i}\right)$, where $L_{i+1}$ is the left-hand side of rule $r_{i+1}$. The sequence graph $\left(m_{0}\right) \Rightarrow_{r_{1}}$ $\operatorname{graph}\left(m_{1}\right) \Rightarrow_{r_{2}} \ldots \Rightarrow_{r_{n}} \operatorname{graph}\left(m_{n}\right)$ together with the morphisms $\varphi_{i}$ is an abstract run. We denote by $\operatorname{Run}_{A}(P, \iota)$ the set of all abstract runs of the Petri $\operatorname{graph}(P, \iota)$.

Each real run $\mathcal{J}_{r}=\left(G_{0} \Rightarrow_{r_{1}} G_{1} \Rightarrow_{r_{2}} \ldots \Rightarrow_{r_{n}} G_{n}\right)$ of the GTS $\left(\mathcal{R}, G_{0}\right)$ can be considered as an abstract run where the $\varphi_{i}: L_{i+1} \rightarrow G_{i}$ represent the matches of the left-hand sides of the rules $r_{i}$.

Proposition 1. Let $\mathcal{C}_{\mathcal{G}}$ be an over-approximation for a GTS $\mathcal{G}$ computed by Algorithm 7. Then, for every real run $\mathcal{J}_{r}$ of the graph transformation system there exists an abstract run $\mathcal{J} \in \operatorname{Run}_{A}\left(\mathcal{C}_{\mathcal{G}}\right)$ such that $\mathcal{J}_{r}$ corresponds to $\mathcal{J}$, i.e., $\mathcal{J}_{r} \lll \mathcal{J}$.

An abstract run $\mathcal{J}$ for which there does not exist a real run corresponding to $\mathcal{J}$ is called spurious. If, at the same time, it violates the property we attempt to verify, it is called a counterexample or error trace.

We can now verify the GTS by analyzing the Petri graph underlying the Petri net. For instance, in order to show that no reachable graph contains a subgraph $G_{s}$ we add a new rule to the GTS with $G_{s}$ as left-hand side and an edge with a new label Error in the right-hand side (see rule "Error" in Table 1). If we can show that either no place labelled Error exists in the net or every such place is not coverable (this can be done using coverability graphs or backward reachability algorithms [1]), then we can deduce that this property holds. 
However, if the approximation is too coarse, we might not be able to verify the property. We have shown in [5] how to construct a sequence of subsequently better unfolding - which however grow in size fairly rapidly - by forbidding folding steps up to depth $k$. Therefore we will now show how to successfully apply the technique of counterexample-guided abstraction refinement in our framework.

\section{Abstraction Refinement}

In order to eliminate spurious runs, we will show that they are always caused by the fact that certain nodes were merged. We will identify these nodes and show how to avoid their being merged in the next iteration, thereby avoiding this particular spurious run and all other abstract runs corresponding to it in a sense made precise later. Merging of nodes is harmful since it might produce new left-hand sides, thereby leading to additional rewriting steps.

\subsection{Spurious Runs}

For a given abstract run $\mathcal{J}=\left(\operatorname{graph}\left(m_{0}\right) \Rightarrow r_{r_{1}} \operatorname{graph}\left(m_{1}\right) \Rightarrow \Rightarrow_{r_{2}} \quad \ldots \Rightarrow_{r_{n}}\right.$ $\left.\operatorname{graph}\left(m_{n}\right)\right)$ of the Petri graph with morphisms $\varphi_{i}: L_{i+1} \rightarrow \operatorname{graph}\left(m_{i}\right)$ we define $\mathcal{H}$ to be the set of real runs corresponding to the prefixes of $\mathcal{J}$. Furthermore let $\mathcal{H}_{i}$ be the set of hypergraphs reachable after $i$ steps in a real run $\mathcal{J}_{r} \in \mathcal{H}$. It holds that $\mathcal{H}_{0}=\left\{G_{0}\right\}$.

An abstract run $\mathcal{J}$ is spurious if $\mathcal{H}_{n}=\emptyset$. If the run is spurious, there exists a $k$ such that $\mathcal{H}_{k} \neq \emptyset$, but $\mathcal{H}_{k+1}=\emptyset$ (and therefore also $\mathcal{H}_{l}=\emptyset$ for $l>k$ ). It will be shown in the following how to construct a new refined over-approximation $\mathcal{C}_{\mathcal{G}}^{\prime}$, which does not contain $\mathcal{J}$ and some other spurious runs corresponding to $\mathcal{J}$.

Example: We illustrate the idea of a spurious abstract run with the run corresponding to the firing of the highlighted transitions "Cross Location" and "Error" in Fig. 3. In fact, there is not real run in the original GTS that corresponds to it.

\subsection{Relations on Nodes for Refining Abstract Runs}

According to Algorithm 7 and Definition 8 it holds that $\mathcal{H}_{k} \neq \emptyset$ and $\mathcal{H}_{k+1}=\emptyset$ if and only if for each $G \in \mathcal{H}_{k}$ there exists no edge-injective morphism $\eta$ : $L_{k+1} \rightarrow G$ such that the following diagram commutes, where $\xi_{k}$ is an edgebijective morphism derived from the correspondence property (see Definition 8). In other words: there is no way to find a match of the left-hand side in $G$ that agrees with the abstract run.

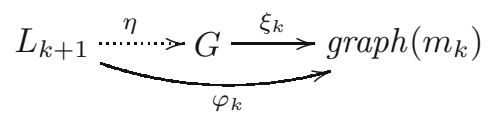

For if there were such a match morphism $\eta$, we could rewrite $G$ to $G^{\prime}$ with rule $r_{k+1}$ corresponding to the transition transforming $m_{k}$ to $m_{k+1}$. Because of 
the construction of the Petri graph, where the right-hand side of $r_{i+1}$ has been attached during an unfolding step, we would then be able to find an edge-bijective morphism $\xi_{k+1}: G^{\prime} \rightarrow \operatorname{graph}\left(m_{k+1}\right)$ thus continuing the correspondence.

Such a situation is only possible if $\xi_{k}$ is non-injective on some nodes of $G$, i.e., these nodes were merged during construction of the over-approximation $\mathcal{C}_{\mathcal{G}}$, which is the reason for the spurious run.

Example: In our running example (see Fig. 11 and 2) the nodes $v_{1}$ and $v_{2}$ as well as $w_{1}$ and $w_{2}$ of the initial hypergraph have been merged by the overapproximation, becoming $v_{1,2}$ and $w_{1,2}$. This led to the spurious abstract run described above.

We will now show how to determine the node merges which caused the spurious run. Consider, for a fixed graph $G$ and a morphism $\xi_{k}$, the set $\Theta$ of possible equivalence relations $\sim$ on nodes for a graph $G \in \mathcal{H}_{k}$ such that, after merging the nodes in each equivalence class, we can find an appropriate match of the left-hand side $L_{k+1}$ in the graph $G / \sim$. More formally, we demand the existence of an edge-injective morphism $\eta^{\prime}: L_{k+1} \rightarrow G / \sim$ such that the following diagram commutes, where $\xi_{k}^{\prime}: G / \sim \rightarrow \operatorname{graph}\left(m_{k}\right)$ is obtained by quotienting $\xi_{k}$ according to $\sim$.

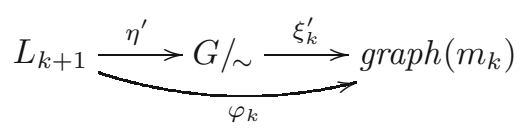

In order to characterize the smallest equivalence in $\Theta$ consider a node $v$ of the left-hand side and determine a set $Q_{v}$ of nodes in $G$ which have to be fused into one node which is the image of $v$ under $\eta^{\prime}$. Let $v \in V_{L_{k+1}}$ and let $e$ be an edge of $L_{k+1}$ with $c_{i}(e)=v$ for some $i$. For every edge $e^{\prime}$ in $G$ with $\xi_{k}\left(e^{\prime}\right)=\varphi_{k}(e)$ we require that $c_{i}\left(e^{\prime}\right) \in Q_{v}$.

Consider the relation $\mathcal{Q}$, where for each $v \in V_{L_{k+1}}$ all nodes in $Q_{v}$ are related and the relation $\widehat{\mathcal{Q}}$ which is the smallest equivalence containing $\mathcal{Q}$.

Proposition 2. The equivalence $\widehat{\mathcal{Q}}$ constructed above is the smallest equivalence contained in $\Theta$.

Example: We consider again the abstract error trace $\mathcal{J}$ which can be obtained by firing transitions "Cross Location" and "Error". However, this error trace has no real runs that correspond to it, which can be seen by computing the set $\mathcal{H}$ of runs corresponding to prefixes of $\mathcal{J}$. Here, the set $\mathcal{H}_{0}$ consists of the initial hypergraph and the set $\mathcal{H}_{1}$ contains one graph $G_{1}$. The next rule "Error" cannot be applied to $G_{1}$ in such a way that the corresponding diagram commutes and therefore the set $\mathcal{H}_{2}$ is empty.

Fig. 4 shows the left-hand side of rule "Error", $G_{1} \in \mathcal{H}_{1}$ and $\operatorname{graph}\left(m_{1}\right)$, the graph corresponding to the marking reached after one step. One notices that no appropriate morphism $\eta$ can be found unless the nodes $w_{1}$ and $w_{2}$ in $G_{1}$ are merged. Therefore we have $Q_{w_{1}^{\prime}}=\left\{w_{1}, w_{2}\right\}, Q_{w_{2}^{\prime}}=\left\{w_{2}\right\}$ and the smallest

$\overline{{ }^{3} \text { Note that by }} c_{i}(e)$ we denote the $i$-the node in the sequence $c(e)$. 


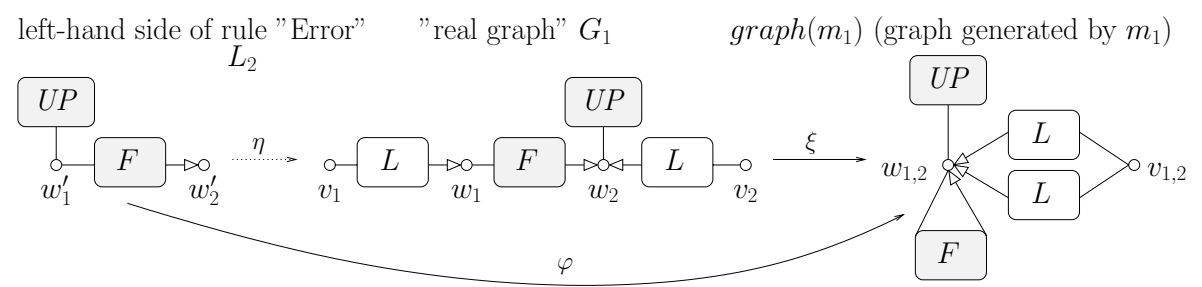

Fig. 4. Hypergraphs $G_{1} \in \mathcal{H}_{1}, L_{2}$ and $\operatorname{graph}\left(m_{1}\right)$ from the firewall example

equivalence relation $\widehat{\mathcal{Q}}$ relates the nodes $w_{1}$ and $w_{2}$ and no other nodes. Note for instance that $w_{2}$ must be contained in $Q_{w_{1}^{\prime}}$ since both are attached to the unary edge labelled $U P$.

\subsection{Elimination of Spurious Runs}

The general idea for destroying spurious runs is to avoid the merging of nodes from the same equivalence class of $\widehat{\mathcal{Q}}$. For this reason we assign colours to the nodes of the graphs contained in $\mathcal{H}$ and disallow the merging of nodes corresponding to nodes with the same colour. For reasons that will become clear below a node may have several colours, i.e., a node $v$ is associated to a set $\operatorname{cols}(v)$ of colours.

For each $G \in \mathcal{H}_{k}$ we and each morphism $\xi_{k}: G \rightarrow \operatorname{graph}\left(m_{k}\right)$ we consider the corresponding relation $\mathcal{Q}_{G, \xi_{k}}$. Then we assign colours to nodes in such a way that there exists at least one pair $v_{1}, v_{2}$ of nodes such that $v_{1} \mathcal{Q}_{G, \xi_{k}} v_{2}$ and $\operatorname{cols}\left(v_{1}\right) \cap \operatorname{cols}\left(v_{2}\right) \neq \emptyset$. There are several ways to do this and all of them will help to eliminate the counterexample. In our implementation we choose a color for each set of nodes $Q_{v}$ and assign it to all nodes contained in $Q_{v}$.

In order to catch "bad" mergings as early as possible, these colours have to be distributed to the remaining graphs contained in $\mathcal{H}$. Let us recall here that according to Definition 3 for each real run $\mathcal{J}_{r}=\left(G_{0} \Rightarrow_{r_{1}} G_{1} \ldots \Rightarrow_{r_{k}} G_{k}\right)$ from $\mathcal{H}$ we have injective partial morphisms $\nu_{i}: G_{i} \rightarrow G_{i+1}$ for $i=0, \ldots, k-1$. Using these partial morphisms we assign the colours of $G_{k}$ to the remaining graphs $G_{i}$ contained in $\mathcal{H}$. We start from $G_{k}$ and proceed as follows: if a node $v \in G_{i+1}$ has a colour then we also assign this colour to the node $\nu^{-1}(v)$ if such a node exists. In this way a node may obtain several colours, due to the branching structure of the runs contained in $\mathcal{H}$. We denote by $\operatorname{cols}(v)$ the set of colours of the node $v \in V_{G_{j}}$ where $G_{j} \in \mathcal{H}_{j}$.

We are now ready to give the algorithm for computing the refined overapproximation.

\section{Algorithm 10 (Refined approximated unfolding).}

Input: A GTS $\mathcal{G}$, a set $\mathcal{H}$ of runs corresponding to prefixes of the counterexample and a function cols assigning sets of colours to the nodes of the graphs in $\mathcal{H}$.

Output: The refined over-approximation $\mathcal{C}_{\mathcal{G}}^{\prime}$. 
We start constructing the new over-approximation $\mathcal{C}_{\mathcal{G}}^{\prime}$ with the initial graph $G_{0}$. Unfolding steps will be performed as described in Algorithm 7.

For a folding step we disallow the merging of nodes corresponding to nodes in $\mathcal{H}$ having the same colour. More specifically, consider the over-approximation $\mathcal{C}_{\mathcal{G}}^{\prime}$, which is currently being constructed. Now for each run $\mathcal{J}_{r}=G_{0} \Rightarrow_{r_{1}} \ldots \Rightarrow_{r_{\ell}} G_{\ell}$ in $\mathcal{H}$ where $\ell<k$ check the following:

We consider all abstract runs $\mathcal{J}=\operatorname{graph}\left(m_{0}\right) \Rightarrow_{r_{1}} \ldots \Rightarrow_{r_{\ell}} \operatorname{graph}\left(m_{\ell}\right)$ of the current Petri graph $\mathcal{C}_{\mathcal{G}}^{\prime}$ for which $\mathcal{J}_{r} \ll \mathcal{J}$ and all edge-bijective morphisms $\xi: G_{i} \rightarrow \operatorname{graph}\left(m_{i}\right)$ for $i=0, \ldots, \ell$. Whenever there are two nodes $v_{1}, v_{2}$ in $G_{i}$ with $\operatorname{cols}\left(v_{1}\right) \cap \operatorname{cols}\left(v_{2}\right) \neq \emptyset$ and $\xi\left(v_{1}\right)=\xi\left(v_{2}\right)$, we have erroneously merged two nodes in the approximation which should not have been merged. Consequently this folding step is undone.

Previously rejected folding steps are recorded and are not any more considered by the algorithm.

In this way we will eliminate not only the spurious run but several more runs which are characterized below (see Proposition 5 ).

Example: Fig. 5 depicts the hypergraph obtained for the firewall example after the abstraction refinement procedure. As one can see, the "critical nodes" of the hypergraph, namely the nodes $w_{1}$ and $w_{2}$, are now separated.

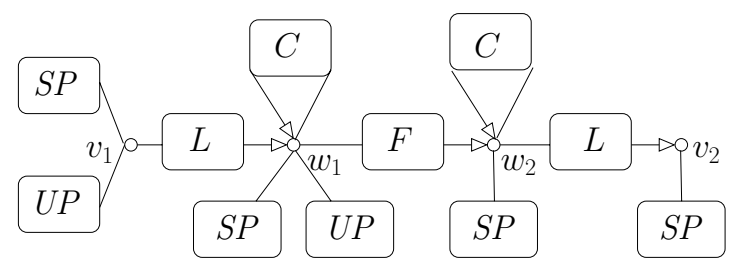

Fig. 5. Hypergraph obtained after abstraction refinement

\subsection{Correctness}

In the following we will show that Algorithm 10 terminates and that the refined over-approximation is correct and more exact then the previous one.

Let $\mathcal{C}_{\mathcal{G}}$ be an over-approximation with a spurious run $\mathcal{J}$ and let $\mathcal{C}_{\mathcal{G}}^{\prime}$ be the corresponding refined over-approximation. In [3] it is shown that the algorithm constructing the over-approximating Petri graph terminates. We modified the algorithm by forbidding some of the folding steps and hence we have to reprove termination for the new version of the algorithm.

Proposition 3. The algorithm computing the refined over-approximation $\mathcal{C}_{\mathcal{G}}^{\prime}$ for a given GTS $\mathcal{G}$ and a (spurious) abstract run $\mathcal{J}$ of $\mathcal{C}_{\mathcal{G}}$ terminates.

Furthermore the new over-approximation is still a valid over-approximation as before. 
Proposition 4. Let $\mathcal{C}_{\mathcal{G}}^{\prime}$ be the refined over-approximation of the GTS. Then, for every real run $\mathcal{J}_{r}$ of the graph transformation system there exists an abstract run $\mathcal{J} \in \operatorname{Run}_{A}\left(\mathcal{C}_{\mathcal{G}}^{\prime}\right)$ such that $\mathcal{J}_{r}$ corresponds to $\mathcal{J}$, i.e., $\mathcal{J}_{r} \lll \mathcal{J}$.

In the following two propositions we will show that we have eliminated the given spurious counterexample and have not added any new ones. First we should answer the following question: what kind of runs have we eliminated by abstraction refinement? It is easy to see that in the refined over-approximation we have lost the initial spurious counter-example $\mathcal{J}$. In fact we have not only eliminated $\mathcal{J}$, but some more runs as described below.

Definition 11 (Correspondence with respect to runs). Let $(P, \iota)$ and $\left(P^{\prime}, \iota^{\prime}\right)$ be two Petri graphs for a GTs $\left(\mathcal{R}, G_{0}\right)$. Furthermore let $\mathcal{J} \in$ Run $_{A}(P, \iota)$ and $\mathcal{J}^{\prime} \in \operatorname{Run}_{A}\left(P^{\prime}, \iota^{\prime}\right)$ be two abstract runs of these Petri graphs and let $\mathcal{H}$ be the set of real runs considered earlier. We say that $\mathcal{J}^{\prime}$ corresponds to $\mathcal{J}$ with respect to $\mathcal{H}$ if $\mathcal{J}^{\prime}$ corresponds to $\mathcal{J}$ and a run $\mathcal{J}^{\prime \prime} \in \mathcal{H}$ of maximal length weakly corresponds to a prefix of $\mathcal{J}^{\prime}$.

Using this definition we can now state and prove the following propositions.

Proposition 5. The refined over-approximation $\mathcal{C}_{\mathcal{G}}^{\prime}$, constructed above does not contain any run $\mathcal{J}^{\prime}$ corresponding to the spurious run $\mathcal{J}$ of $\mathcal{C}_{\mathcal{G}}$ with respect to $\mathcal{H}$.

We can also show that no new spurious runs have appeared, which means that the new approximation is strictly better than the old one.

Proposition 6. If the refined over-approximation $\mathcal{C}_{\mathcal{G}}^{\prime}$ contains a spurious run $\mathcal{J}^{\prime}$, then it corresponds to some spurious run $\mathcal{J}$ in $\mathcal{C}_{\mathcal{G}}$.

We remark that the considered abstraction refinement approach can also be implemented in the case of any number of spurious counterexamples by iteratively refining the abstraction. Naturally, due to undecidability and the fact that GTSs are in general Turing-complete, there is no guarantee that it will ever terminate.

\section{$5 \quad$ Implementation and Experimental Results}

In this section we consider examples of GTSs and compare the experimental results obtained by refining the approximation by forbidding folding steps up to a certain depth (see [5]) and counterexample-guided abstraction refinement as presented in this paper. It is shown that for practical purposes the new technique is usually more efficient.

The algorithm was implemented in $\mathrm{C}++$ under Linux and the computer parameters are $2 *$ Xeon $2.4 \mathrm{GHz}, 2 \mathrm{~GB}$ RAM.

For case studies we have chosen two distributed systems: the running example of this paper (firewall example) and a system of public and private servers (for a description of the second example see [11]). If we compare the results in 
Table 2. Verification results (abstraction refinement by forbidding folding steps up to a certain depth $k$, i.e., by computing $k$-coverings)

\begin{tabular}{|c|c|c|c|c|c|c|}
\hline example & $k$ (depth) & nodes & edges & transitions & time (sec) & verified \\
\hline \hline Public/private servers I & 0 & 1 & 9 & 13 & 0.05 & no \\
\hline Public/private servers I & 1 & 2 & 19 & 34 & $\mathbf{0 . 7 2}$ & yes \\
\hline \hline Public/private servers II & 0 & 1 & 10 & 14 & 0.05 & no \\
\hline Public/private servers II & 1 & 1 & 11 & 16 & 0.07 & no \\
\hline Public/private servers II & 2 & 3 & 31 & 63 & $\mathbf{7 . 1 6}$ & yes \\
\hline \hline Firewall I & 0 & 2 & 8 & 13 & 0.05 & no \\
\hline Firewall I & 1 & 6 & 25 & 50 & 2.4 & no \\
\hline Firewall I & 2 & 10 & 51 & 148 & 138.18 & no \\
\hline \hline Firewall II & 0 & 2 & 8 & 13 & 0.14 & no \\
\hline Firewall II & 1 & 8 & 39 & 82 & 13.7 & no \\
\hline Firewall II & 2 & 14 & 79 & 242 & 858.4 & no \\
\hline
\end{tabular}

Table 3. Verification results (counterexample-guided abstraction refinement)

\begin{tabular}{|c|c|c|c|c|c|}
\hline example & nodes & edges & transitions & time $(\mathrm{sec})$ & verified \\
\hline \hline Public/private servers I & 2 & 16 & 25 & $\mathbf{0 . 6 7}$ & yes \\
\hline \hline Public/private servers II & 2 & 17 & 26 & $\mathbf{0 . 6 8}$ & yes \\
\hline \hline Firewall I & 4 & 11 & 17 & $\mathbf{0 . 1 6}$ & yes \\
\hline \hline Firewall II & 4 & 12 & 18 & $\mathbf{0 . 3 3}$ & yes \\
\hline
\end{tabular}

Tables 2 and 3 it can be seen that in the case of counterexample-guided abstraction refinement we have an advantage both in runtime for computing the approximation and in the size of the over-approximations, which are consequently easier to analyze. The difference is especially pronounced for versions II, which use larger start graphs.

The efficiency of the abstraction refinement approach can be explained by the fact that we forbid to merge only those parts of the unfolding which are responsible for the spurious counterexample. This means that the over-approximation remains rather compact compared to the depth-based (or $k$-covering) approach, where we are not allowed to merge all items having depth smaller than $k$. Note that for the firewall example it was not possible to verify the properties using the depth-based approached.

\section{Conclusion}

In this paper we have shown how counterexample-guided abstraction refinement can be applied to the analysis of dynamically evolving graphical structures in a fully automatic way. In this case we are not concerned with the abstraction of data values, but rather with graphs that are abstracted by merging nodes 
and edges, using the concept of graph morphisms. Hence, abstraction refinement can in this case be described by exploiting commutativity or rather noncommutativity of morphisms as described in Section 4. Also, since we are dealing with the approximation of graph structures rather than data values, no theorem prover is needed in order to determine the initial abstraction, instead we use techniques for approximated unfolding developed in [3].

Apart from smaller case studies we have used our approximated unfolding technique to verify a mutual exclusion protocol [9] and to verify insertion of elements into red-black trees [2]. We are currently working on an encoding of simple pointer programs into graph rewriting which will enable us to directly verify operations on pointer structures.

Research concerned with the verification of dynamically evolving graph structures which can be used to model distribution and mobility is fairly recent. There are contributions coming from the area of dataflow analysis such as shape analysis [16] as well as work directed more specifically towards the analysis of graph transformation systems [14, 13, 17, 9]. We believe that introducing counterexample-based abstraction refinement is an important step in order to make such verification techniques usable in practice. We also think that some of the techniques presented here can be employed in fairly general settings.

Compared to shape analysis [16, 12] which is also concerned with over-approximation techniques for graphical structures and which represents these structures as models of a 3-valued logic, we follow a different approach where graphs are represented directly and graph morphisms are used as a convenient abstraction mechanism. Furthermore we approximate with Petri nets, which enable us to talk about multiplicities of edges and can be conveniently analyzed using a variety of existing Petri net tool.

Acknowledgments. We would like to thank Tobias Heindel, Paolo Baldan and Andrea Corradini for many interesting discussions on the topics of this paper.

\section{References}

1. P.A. Abdulla, B. Jonsson, M. Kindahl, and D. Peled. A general approach to partial order reductions in symbolic verification. In Proc. of CAV '98, pages 379-390. Springer, 1998. LNCS 1427.

2. P. Baldan, A. Corradini, J. Esparza, T. Heindel, B. König, and V. Kozioura. Verifying red-black trees. In Proc. of COSMICAH '05, 2005. Proceedings available as report RR-05-04 (Queen Mary, University of London).

3. P. Baldan, A. Corradini, and B. König. A static analysis technique for graph transformation systems. In Proc. of CONCUR '01, pages 381-395. Springer, 2001. LNCS 2154.

4. P. Baldan, A. Corradini, and B. König. Static analysis of distributed systems with mobility specified by graph grammars - a case study. In Proc. of IDPT '02. Society for Design and Process Science, 2002.

5. P. Baldan and B. König. Approximating the behaviour of graph transformation systems. In Proc. of ICGT '02, pages 14-29. Springer, 2002. LNCS 2505. 
6. T. Ball and S.K. Rajamani. Automatically validating temporal safety properties of interfaces. In Proc. of SPIN '01, pages 103-122. Springer, 2001. LNCS 2057.

7. S. Chaki, E. Clarke, A. Groce, S. Jha, and H. Veith. Modular verification of software components in C. In Proc. of ICSE '03, pages 385-395. IEEE Computer Society, 2003.

8. E. Clarke, S. Grumberg, S. Jha, Y. Lu, and H. Veith. Counterexample-guided abstraction refinement. In Proc. of $C A V$ '00, pages 154-169. Springer, 2000. LNCS 1855 .

9. F.L. Dotti, L. Foss, L. Ribeiro, and O. Marchi Santos. Verification of distributed object-based systems. In Proc. of FMOODS '03, pages 261-275. Springer, 2003. LNCS 2884.

10. T.A. Henzinger, R. Jhala, R. Majumdar, and G. Sutre. Lazy abstraction. In Proc. of POPL '02, pages 58-70. ACM, 2002.

11. Barbara König and Vitali Kozioura. Counterexample-guided abstraction refinement for the analysis of graph transformation systems. Technical Report 01/2006, Universität Stuttgart, 2006.

12. A. Loginov, T. Reps, and M. Sagiv. Abstraction refinement via inductive learning. In Proc. of $C A V$ '05, pages 519-533. Springer, 2005. LNCS 3576.

13. A. Rensink. Canonical graph shapes. In Proc. of ESOP '04, pages 401-415. Springer, 2004. LNCS 2986.

14. A. Rensink. State space abstraction using shape graphs. In Proc. of AVIS '04, ENTCS, 2004. to appear.

15. G. Rozenberg, editor. Handbook of Graph Grammars and Computing by Graph Transformation, Vol. 1: Foundations. World Scientific, 1997.

16. M. Sagiv, T. Reps, and R. Wilhelm. Parametric shape analysis via 3 -valued logic. TOPLAS, 24(3):217-298, 2002.

17. D. Varró. Towards symbolic analysis of visual modeling languages. In Proc. of GT-VMT' '02, volume 72 of ENTCS. Elsevier, 2002. 